\title{
ABOUT THE TWO-STAGE PROXIMAL METHOD FOR SOLVING OF EQUILIBRIUM PROBLEMS
}

\author{
Ya. I. Vedel ${ }^{1}$, V. V. Semenov ${ }^{1}$, L. M. Chabak ${ }^{2}$ \\ ${ }^{1}$ Faculty of Computer Science and Cybernetics, Taras Shevchenko Kiev National University, \\ Kiev, Ukraine, E-mail: \{yana.vedel, semenov.volodya\}@gmail.com \\ ${ }^{2}$ Faculty of Management and Technology, Infrastructure and Technology State University, \\ Kiev, Ukraine, E-mail: chabaklm@ukr.net
}

\section{О ДВУХЭТАПНОМ ПРОКСИМАЛЬНОМ АЛГОРИТМЕ ДЛЯ РЕШЕНИЯ ЗАДАЧИ О РАВНОВЕСИИ}

\author{
Я. И. ВеДЕЛЬ ${ }^{1}$, В. В. Семёнов ${ }^{1}$, Л. М. ЧАБАК ${ }^{2}$ \\ ${ }^{1}$ Факультет компьютерных наук и кибернетики, Киевский национальный университет \\ имени Тараса Шевченко, Киев, Украина, E-mail: \{yana.vedel, semenov.volodya\}@gmail.com \\ ${ }^{2}$ Факультет управления и технологий, Государственный институт инфраструктуры и \\ технологий, Киев, Украина, E-mail: chabaklm@ukr.net
}

ABSTRACT. In this paper, the weak convergence of an iterative twostage proximal method for the approximate solution of the equilibrium problem in a Hilbert space is investigated. This method was recently been developed by Vedel and Semenov and can be used to solve mathematical programming problems, variational inequalities and game theory problems. The analysis of the convergence of the method was carried out under the assumption of the existence of a solution of the equilibrium problem and under conditions weaker than the previously considered ones.

KEYWORDS: equilibrium problem, two-stage proximal method, bifunction, pseudo-monotonicity, Lipschitz condition, Hilbert space, weak convergence.

АннотАция. В работе исследована слабая сходимость итерационного двухэтапного проксимального метода приближенного решения задачи о равновесии в гильбертовом пространстве. Данный метод был недавно разработан Я. И. Ведель и В. В. Семёновым и может быть использован для решения задач математического программирования, вариационных неравенств и игровых задач. Анализ сходимости метода проведен в предположении о существовании решения задачи о равновесии и при условиях более слабых, чем ранее рассмотренные.

КЛЮчЕВЫЕ СЛОВА: Задача о равновесии, двухэтапный проксимальный метод, бифункция, псевдомонотонность, липшицевость, гильбертово пространство, слабая сходимость. 


\section{ВВеДЕНИЕ}

Важным и весьма популярным направлением современного прикладного нелинейного анализа является исследование задач о равновесии (неравенств Ки Фаня, задач равновесного программирования) вида [1-5]

$$
\text { найти } x \in C: F(x, y) \geq 0 \quad \forall y \in C \text {, }
$$

где $C$ - непустое подмножество гильбертова пространства $H, F: C \times C \rightarrow$ $\mathbb{R}$ - бифункция. В виде (1) можно сформулировать задачи математического программирования, вариационные неравенства и многие игровые задачи. Приведем три типичные формулировки.

(1) Если $F(x, y)=\varphi(y)-\varphi(x)$, где $\varphi: C \rightarrow \mathbb{R}$, то задача (1) является задачей условной минимизации

$$
\varphi \rightarrow \min _{C} .
$$

(2) Если $F(x, y)=(A x, y-x)$, где $A: C \rightarrow H$, то задача (1) сводится к классическому вариационному неравенству

$$
\text { найти } x \in C:(A x, y-x) \geq 0 \forall y \in C \text {. }
$$

(3) Пусть $I-$ конечное множество индексов. Для каждого $i \in I$ заданы множество $C_{i}$ и функция $\varphi_{i}: C \rightarrow \mathbb{R}$, где $C=\prod_{i \in I} C_{i}$. Для $x=$ $\left(x_{i}\right)_{i \in I} \in C$ обозначим $x^{i}=\left(x_{j}\right)_{j \in I, j \neq i}$. Точку $\bar{x}=\left(\bar{x}_{i}\right)_{i \in I}$ называют равновесием Нэша, если для всех $i \in I$ выполняются неравенства

$$
\varphi_{i}(\bar{x}) \leq \varphi_{i}\left(\bar{x}^{i}, y_{i}\right) \quad \forall y_{i} \in C_{i} .
$$

Определим функцию $F: C \times C \rightarrow \mathbb{R}$ следующим образом

$$
F(x, y)=\sum_{i \in I}\left(\varphi_{i}\left(x^{i}, y_{i}\right)-\varphi_{i}(x)\right) .
$$

Точка $\bar{x} \in C$ является равновесием Нэша тогда и только тогда, когда она является решением задачи (1).

Алгоритмам решения равновесных и близких задач посвящено большое количество работ. Частным случаем задач о равновесии являются вариационные неравенства. Для их решения Г. М. Корпелевич предложила экстраградиентный метод [6]. Аналогам экстраградиентного метода для задач о равновесии посвящены работы $[1,5,7]$.

В 1980 Л. Д. Попов [8] предложил для поиска седловых точек выпукловогнутых функций, определенных в конечномерном евклидовом пространстве, интересную модификацию метода Эрроу-Гурвица. В статье [9] был предложен двухэтапный проксимальный алгоритм для решения задач о равновесии в гильбертовом пространстве, являющийся адаптацией метода Л. Д. Попова к общим задачам равновесного программирования (см. также $[10,11])$.

В данной работе уделено внимание теоретическому анализу двухэтапного алгоритма из [9]. А именно, доказана его слабая сходимость при более слабых, чем в [9], предположениях. 


\section{1. ПОСТАНОВКА ЗАДАЧИ О РАВНОВЕСИИ И ОПИСАНИЕ АЛГОРИТМА}

Всюду далее $H$ - действительное гильбертово пространство со скалярным произведением $(\cdot, \cdot)$ и порожденной нормой $\|\cdot\|$. Сильную и слабую сходимость в $H$ последовательности $\left(x_{n}\right)$ к $x$ обозначим $\rightarrow$ и $\rightarrow$, соответственно.

Пусть $g: H \rightarrow \mathbb{R} \cup\{+\infty\}$ - собственная выпуклая полунепрерывная снизу функция. Напомним, что проксимальным оператором [12], ассоциированным с функцией $g$, называют оператор

$$
H \ni x \mapsto \operatorname{prox}_{g} x=\operatorname{argmin}_{y \in \operatorname{dom} g}\left(g(y)+\frac{1}{2}\|y-x\|^{2}\right) \in \operatorname{dom} g .
$$

Оператор $\operatorname{prox}_{g}$ - твердо нерастягивающий (firmly nonexpansive) и

$$
g(y)-g(z)+(z-x, y-z) \geq 0 \forall y \in \operatorname{dom} g \Leftrightarrow z=\operatorname{prox}_{g} x .
$$

Для непустого выпуклого замкнутого множества $C \subseteq H$ и бифункции $F: C \times C \rightarrow \mathbb{R}$ рассмотрим задачу о равновесии:

$$
\text { найти } x \in C: F(x, y) \geq 0 \quad \forall y \in C \text {. }
$$

Будем предполагать выполненными следующие условия:

(A1) $F(x, x)=0$ для всех $x \in C$;

(A2) для всех $x, y \in C$ из $F(x, y) \geq 0$ следует $F(y, x) \leq 0$ (псевдомонотонность);

(А3) для всех $x \in C$ функция $F(x, \cdot)$ полунепрерывна снизу и выпукла на $C$;

(A4) для всех $y \in C$ функция $F(\cdot, y)$ полунепрерывна сверху на $C$;

(A5) для всех $x, y, z \in C$ имеет место

$$
F(x, y) \leq F(x, z)+F(z, y)+a\|x-z\|^{2}+b\|z-y\|^{2},
$$

где $a, b-$ положительные константы (липшицевость).

Замечание 1. Условие (А5) типа липшицевости введено G. Mastroeni в [2]. Например, бифункция $F(x, y)=(A x, y-x)$ с липшицевым оператором $A: C \rightarrow H$ удовлетворяет (А5) с $a=b=\frac{L}{2}$.

Рассмотрим так называемую дуальную [4] (для задачи (3)) задачу о равновесии:

$$
\text { найти } x \in C: F(y, x) \leq 0 \quad \forall y \in C \text {. }
$$

Множества решений задач (3) и (4) обозначим $S$ и $S^{*}$. При выполнении условий (A1)-(А4) имеем $S=S^{*}$ [4]. В частности, множество $S-$ выпукое и замкнутое, поскольку

$$
S=S^{*}=\bigcap_{y \in C}\{x \in C: F(y, x) \leq 0\} .
$$

Далее будем предполагать, что $S \neq \emptyset$.

Для приближенного решения задачи (3) рассмотрим следующий итерационный 
Алгоритм 1 (Я. И. Ведель, В. В. Семенов, [9]). Для $x_{1}, y_{0} \in C$ генерируем последовательность элементов $x_{n}, y_{n} \in C$ при помощи итерационной схемы

где $\lambda_{n}>0$.

$$
\left\{\begin{array}{l}
y_{n}=\operatorname{prox}_{\lambda_{n} F\left(y_{n-1}, \cdot\right)} x_{n}, \\
x_{n+1}=\operatorname{prox}_{\lambda_{n} F\left(y_{n}, \cdot\right)} x_{n},
\end{array}\right.
$$

На каждом шаге алгоритма 1 следует решить две выпуклые задачи с сильно выпуклыми функциями. Предположим возможность их эффективного решения. Алгоритм 1 был предложен в работе [9] (см. также [10,11]). В настоящей работе алгоритм 1 рассматривается при более слабых предположениях.

Замечание 2. Если $F(x, y)=(A x, y-x)$, то алгоритм 1 принимает вид:

$$
\left\{\begin{array}{l}
x_{1} \in C, y_{0} \in C, \\
y_{n}=P_{C}\left(x_{n}-\lambda_{n} A y_{n-1}\right), \\
x_{n+1}=P_{C}\left(x_{n}-\lambda_{n} A y_{n}\right),
\end{array}\right.
$$

где $P_{C}$ - оператор метрического проектирования на множество $C$.

Частный случай схемы из замечания 2 предложен российским математиком Л. Д. Поповым [8] для поиска седловых точек выпукло-вогнутых функций, определенных в конечномерном евклидовом пространстве. В работе [13] Ю. В. Малицкий и В. В. Семенов доказали сходимость этого алгоритма для неравенств с монотонными и липшицевыми операторами, действующими в бесконечномерном гильбертовом пространстве, а также предложили его модификацию. А в работах [14-16] предложены варианты метода с использованием брэгмановского расстояния вместо евклидового. Заметим, что в последнее время данный метод стал известен в среде специалистов по машинному обучению под названием «Extrapolation from the Past» [17].

Относительно обоснования сходимости алгоритма 1 заметим, что при выполнении для некоторого $n \in \mathbb{N}$ равенств

$$
y_{n}=y_{n-1}=x_{n} \quad \text { или } \quad x_{n+1}=x_{n}=y_{n}
$$

имеет место включение $y_{n} \in S$. Действительно, равенство

$$
x_{n+1}=\operatorname{prox}_{\lambda_{n} F\left(y_{n}, \cdot\right)} x_{n}
$$

означает

$$
F\left(y_{n}, y\right)-F\left(y_{n}, x_{n+1}\right)+\frac{\left(x_{n+1}-x_{n}, y-x_{n+1}\right)}{\lambda_{n}} \geq 0 \forall y \in C .
$$

Из второго равенства (5) следует

$$
F\left(y_{n}, y\right) \geq 0 \forall y \in C
$$

то есть $y_{n} \in S$.

Аналогично, из

$$
F\left(y_{n-1}, y\right)-F\left(y_{n-1}, y_{n}\right)+\frac{\left(y_{n}-x_{n}, y-y_{n}\right)}{\lambda_{n}} \geq 0 \forall y \in C
$$


при первом равенстве в (5) получаем $y_{n} \in S$.

Замечание 3. Вышеприведенное наблюдение просто непосредственное следствие критерия:

$$
x \in S \Leftrightarrow x=\operatorname{prox}_{\lambda F(x, \cdot)} x, \lambda>0 .
$$

Далее будем предполагать, что для всех номеров $n \in \mathbb{N}$ условие (5) не имеет места.

\section{2. ОсHовHOE HePABEHCTBO}

Доказательство сходимости алгоритма 1 начнем с доказательства важного неравенства для порождаемых им последовательностй $\left(x_{n}\right)$ и $\left(y_{n}\right)$.

Лемма 1. Для порожденных алгоритмом 1 последовательностей $\left(x_{n}\right)$, $\left(y_{n}\right)$ и элемента $z \in S$ выполняется неравенство

$$
\begin{aligned}
\left\|x_{n+1}-z\right\|^{2} \leq\left\|x_{n}-z\right\|^{2}-\left(1-2 \lambda_{n} b\right)\left\|x_{n+1}-y_{n}\right\|^{2}- \\
-\left(1-4 \lambda_{n} a\right)\left\|y_{n}-x_{n}\right\|^{2}+4 \lambda_{n} a\left\|x_{n}-y_{n-1}\right\|^{2} .
\end{aligned}
$$

Доказательство. Имеем

$$
\begin{aligned}
&\left\|x_{n+1}-z\right\|^{2}=\left\|x_{n}-z\right\|^{2}-\left\|x_{n}-x_{n+1}\right\|^{2}+2\left(x_{n+1}-x_{n}, x_{n+1}-z\right)= \\
&=\left\|x_{n}-z\right\|^{2}-\left\|x_{n}-y_{n}\right\|^{2}-\left\|y_{n}-x_{n+1}\right\|^{2}- \\
& \quad-2\left(x_{n}-y_{n}, y_{n}-x_{n+1}\right)+2\left(x_{n+1}-x_{n}, x_{n+1}-z\right) .
\end{aligned}
$$

Из определения точек $x_{n+1}$ и $y_{n}$ следует

$$
\begin{gathered}
\lambda_{n} F\left(y_{n}, z\right)-\lambda_{n} F\left(y_{n}, x_{n+1}\right) \geq\left(x_{n+1}-x_{n}, x_{n+1}-z\right), \\
\lambda_{n} F\left(y_{n-1}, x_{n+1}\right)-\lambda_{n} F\left(y_{n-1}, y_{n}\right) \geq-\left(x_{n}-y_{n}, y_{n}-x_{n+1}\right) .
\end{gathered}
$$

Использовав неравенства (8), (9) для оценки скалярных произведений в (7), получаем

$$
\begin{aligned}
\left\|x_{n+1}-z\right\|^{2} \leq\left\|x_{n}-z\right\|^{2}-\left\|x_{n}-y_{n}\right\|^{2}-\left\|y_{n}-x_{n+1}\right\|^{2}+ \\
+2 \lambda_{n}\left\{F\left(y_{n}, z\right)-F\left(y_{n}, x_{n+1}\right)+F\left(y_{n-1}, x_{n+1}\right)-F\left(y_{n-1}, y_{n}\right)\right\} .
\end{aligned}
$$

Из псевдомонотонности бифункции $F$ и включения $z \in S$ следует

$$
F\left(y_{n}, z\right) \leq 0,
$$

а липшицевость $F$ гарантирует выполнение неравенства

$$
\begin{aligned}
-F\left(y_{n}, x_{n+1}\right)+F\left(y_{n-1}, x_{n+1}\right)- & F\left(y_{n-1}, y_{n}\right) \leq \\
& \leq a\left\|y_{n-1}-y_{n}\right\|^{2}+b\left\|y_{n}-x_{n+1}\right\|^{2} .
\end{aligned}
$$

Использовав вышеприведенные оценки в (10), получаем

$$
\begin{aligned}
\left\|x_{n+1}-z\right\|^{2} \leq\left\|x_{n}-z\right\|^{2}- & \left\|x_{n}-y_{n}\right\|^{2}-\left\|y_{n}-x_{n+1}\right\|^{2}+ \\
& +2 \lambda_{n} a\left\|y_{n-1}-y_{n}\right\|^{2}+2 \lambda_{n} b\left\|y_{n}-x_{n+1}\right\|^{2} .
\end{aligned}
$$


Член $\left\|y_{n-1}-y_{n}\right\|^{2}$ оценим следующим образом

$$
\left\|y_{n-1}-y_{n}\right\|^{2} \leq 2\left\|y_{n-1}-x_{n}\right\|^{2}+2\left\|y_{n}-x_{n}\right\|^{2} .
$$

Учтя эту оценку в (12), приходим к неравенству

$$
\begin{aligned}
\left\|x_{n+1}-z\right\|^{2} & \leq\left\|x_{n}-z\right\|^{2}-\left\|x_{n}-y_{n}\right\|^{2}-\left\|y_{n}-x_{n+1}\right\|^{2}+ \\
& +4 \lambda_{n} a\left\|y_{n-1}-x_{n}\right\|^{2}+4 \lambda_{n} a\left\|y_{n}-x_{n}\right\|^{2}+2 \lambda_{n} b\left\|y_{n}-x_{n+1}\right\|^{2},
\end{aligned}
$$

то есть к неравенству (6).

\section{3. СХодимость АЛгоритма}

Перейдем непосредственно к доказательству слабой сходимости алгоритма 1.

Нам потребуются следующие факты.

Лемма 2. Пусть неотрицательные последовательности $\left(a_{n}\right),\left(b_{n}\right)$ таковы, чmo

$$
a_{n+1} \leq a_{n}-b_{n} .
$$

Тогда существует предел $\lim _{n \rightarrow \infty} a_{n} \in \mathbb{R} u \sum_{n=1}^{\infty} b_{n}<+\infty$.

Лемма 3 (Z. Opial, [18]). Пусть последовательность $\left(x_{n}\right)$ элементов гильбертова пространства $H$ слабо сходится $к$ элементу $x \in H$. Тогда для всех $y \in H \backslash\{x\}$ имеем

$$
\liminf _{n \rightarrow \infty}\left\|x_{n}-x\right\|<\liminf _{n \rightarrow \infty}\left\|x_{n}-y\right\| .
$$

Пусть $z \in S$. Положим

$$
\begin{aligned}
& a_{n}=\left\|x_{n}-z\right\|^{2}+4 \lambda_{n} a\left\|y_{n-1}-x_{n}\right\|^{2}, \\
& b_{n}=\left(1-4 \lambda_{n} a\right)\left\|y_{n}-x_{n}\right\|^{2}+\left(1-4 \lambda_{n} a-2 \lambda_{n} b\right)\left\|y_{n}-x_{n+1}\right\|^{2} .
\end{aligned}
$$

Тогда (6) принимает вид

$$
a_{n+1} \leq a_{n}-b_{n} .
$$

Потребуем выполнения условия

$$
0<\underline{\lambda} \leq \lambda_{n} \leq \bar{\lambda}<\frac{1}{2(2 a+b)} .
$$

Тогда из леммы 2 можем сделать вывод, что существует предел

$$
\lim _{n \rightarrow \infty}\left(\left\|x_{n}-z\right\|^{2}+4 \lambda_{n} a\left\|y_{n-1}-x_{n}\right\|^{2}\right)
$$

и

$$
\lim _{n \rightarrow \infty}\left(\left(1-4 \lambda_{n} a\right)\left\|y_{n}-x_{n}\right\|^{2}+\left(1-4 \lambda_{n} a-2 \lambda_{n} b\right)\left\|y_{n}-x_{n+1}\right\|^{2}\right)=0 .
$$

Откуда получаем

$$
\lim _{n \rightarrow \infty}\left\|y_{n}-x_{n}\right\|=\lim _{n \rightarrow \infty}\left\|y_{n}-x_{n+1}\right\|=\lim _{n \rightarrow \infty}\left\|x_{n}-x_{n+1}\right\|=0
$$

и сходимость числовых последовательностей $\left(\left\|x_{n}-z\right\|\right),\left(\left\|y_{n}-z\right\|\right)$ для всех $z \in S$. В частности, последовательности $\left(x_{n}\right),\left(y_{n}\right)$ ограничены. 
Из (8), (9), (10), (11) и (13) следует

$$
\begin{aligned}
& \limsup _{n \rightarrow \infty} F\left(y_{n}, x_{n+1}\right) \leq 0, \\
& \limsup _{n \rightarrow \infty}\left(F\left(y_{n-1}, y_{n}\right)-F\left(y_{n-1}, x_{n+1}\right)\right) \leq 0, \\
& \lim _{n \rightarrow \infty}\left(F\left(y_{n}, x_{n+1}\right)+F\left(y_{n-1}, y_{n}\right)-F\left(y_{n-1}, x_{n+1}\right)\right)=0 .
\end{aligned}
$$

Откуда, в частности, получаем

$$
\lim _{n \rightarrow \infty} F\left(y_{n}, x_{n+1}\right)=0 .
$$

Рассмотрим подпоследовательность $\left(x_{n_{k}}\right)$, слабо сходящуюся к некоторой точке $\bar{z} \in C$. Тогда из (13) следует, что и $y_{n_{k}} \rightarrow \bar{z}$. Покажем, что $\bar{z} \in S$. Имеем

$$
F\left(y_{n}, y\right) \geq F\left(y_{n}, x_{n+1}\right)+\frac{\left(x_{n+1}-x_{n}, x_{n+1}-y\right)}{\lambda} \quad \forall y \in C .
$$

Совершив предельный переход в (15) с учетом (13), (14) и условия (А4), получим

$$
\begin{aligned}
F(\bar{z}, y) & \geq \limsup _{k \rightarrow \infty} F\left(y_{n_{k}}, y\right) \geq \\
& \geq \lim _{k \rightarrow \infty}\left\{F\left(y_{n_{k}}, x_{n_{k}+1}\right)+\frac{\left(x_{n_{k}+1}-x_{n_{k}}, x_{n_{k}+1}-y\right)}{\lambda}\right\}=0 \quad \forall y \in C,
\end{aligned}
$$

то есть $\bar{z} \in S$.

Покажем теперь, что $x_{n} \rightarrow \bar{z}$. Тогда из $x_{n}-y_{n} \rightarrow 0$ следует, что и последовательность $\left(y_{n}\right)$ слабо сходится $\bar{z}$. Рассуждаем от противного. Пусть существует подпоследовательность $\left(x_{m_{k}}\right)$ такая, что $x_{m_{k}} \rightarrow \tilde{z}$ и $\tilde{z} \neq \bar{z}$. Ясно, что $\tilde{z} \in S$. Применим дважды лемму 3 . Имеем

$$
\begin{aligned}
\lim _{n \rightarrow \infty}\left\|x_{n}-\bar{z}\right\|=\lim _{k \rightarrow \infty}\left\|x_{n_{k}}-\bar{z}\right\|<\lim _{k \rightarrow \infty}\left\|x_{n_{k}}-\tilde{z}\right\|=\lim _{n \rightarrow \infty}\left\|x_{n}-\tilde{z}\right\|= \\
\quad=\lim _{k \rightarrow \infty}\left\|x_{m_{k}}-\tilde{z}\right\|<\lim _{k \rightarrow \infty}\left\|x_{m_{k}}-\bar{z}\right\|=\lim _{n \rightarrow \infty}\left\|x_{n}-\bar{z}\right\|,
\end{aligned}
$$

что невозможно. Следовательно, $x_{n} \rightarrow \bar{z}$.

Таким образом, имеет место

Теорема 1. Пусть $H-$ гильбертово пространство, $C \subseteq H-$ непустое выпуклое замкнутое множество, для бифункиии $F: C \times C \rightarrow \mathbb{R}$ выполнены условия (A1)-(A5) и $S \neq \emptyset$. Предположим, что

$$
\lambda_{n} \in[\underline{\lambda}, \bar{\lambda}] \subseteq\left(0, \frac{1}{2(2 a+b)}\right) .
$$

Тогда порожденные алгоритмом 1 последовательности $\left(x_{n}\right),\left(y_{n}\right)$ слабо сходятся к решению $\bar{z} \in S$ задачи о равновесии (3), причем

$$
\lim _{n \rightarrow \infty}\left\|x_{n}-y_{n}\right\|=0 \text {. }
$$

Замечание 4. Теорема 1 обобщает результаты работ $[9,10]$. В этих работах слабая сходимость алгоритма 1 (со стационарной последовательностью $\left.\left(\lambda_{n}\right)\right)$ была доказана при (A1)-(A5) и условии: для ограниченных последовательностей $\left(x_{n}\right),\left(y_{n}\right)$ из С имеет место

$$
\left\|x_{n}-y_{n}\right\| \rightarrow 0 \Rightarrow F\left(x_{n}, y_{n}\right) \rightarrow 0 \text {. }
$$




\section{ЗАКЛЮчЕНИЕ}

В работе получен новый результат о сходимости итерационного двухэтапного проксимального метода решения задач о равновесии в гильбертовом пространстве. Метод был предложен в работе [9] и является развитием модификации Л. Д. Попова [8] схемы Эрроу-Гурвица поиска седловых точек выпукло-вогнутых функций.

Работа выполнена при финансовой поддержке $\mathrm{MOH}$ Украины (проект «Математичне моделювання та оптимізація динамічних систем для оборони, медицини та екології», 0219U008403).

\section{ЛитерАтУрА}

1. Antipin A. S. Equilibrium programming: Proximal methods. Comput. Math. Math. Phys. 1997. Vol. 37. P. 1285-1296.

2. Mastroeni G. On auxiliary principle for equilibrium problems. In: Daniele, P. et al. (eds.) Equilibrium Problems and Variational Models. Kluwer Academic Publishers, Dordrecht, 2003. P. 289-298.

3. Combettes P. L., Hirstoaga S. A. Equilibrium Programming in Hilbert Spaces. J. Nonlinear Convex Anal. 2005. Vol. 6. P. 117--136.

4. Konnov I. V. Application of the proximal point method to nonmonotone equilibrium problems. J. Optim. Theory Appl. 2003. Vol. 119. P. 317-333.

5. Quoc T. D., Muu L. D., Hien N. V. Extragradient algorithms extended to equilibrium problems. Optimization. 2008. Vol. 57. P. 749-776.

6. Korpelevich G. M. The extragradient method for finding saddle points and other problems. Ekonomika i Matematicheskie Metody. 1976. Vol. 12. P. 747-756.

7. Lyashko S. I., Semenov V. V., Voitova T. A. Low-cost modification of Korpelevich's methods for monotone equilibrium problems. Cybernetics and Systems Analysis. 2011. Vol. 47. P. 631-639.

8. Popov L. D. A modification of the Arrow-Hurwicz method for search of saddle points. Mathematical notes of the Academy of Sciences of the USSR. 1980. Vol. 28. Issue 5. P. 845-848.

9. Vedel Y. I., Semenov V. V. A new two-phase proximal method of solving the problem of equilibrium programming. Journal of Computational and Applied Mathematics. 2015. No. 1 (118). P. 15-23.

10. Lyashko S. I., Semenov V. V. A New Two-Step Proximal Algorithm of Solving the Problem of Equilibrium Programming. In: B. Goldengorin (ed.) Optimization and Its Applications in Control and Data Sciences. Springer Optimization and Its Applications, vol. 115. Springer, Cham, 2016. P. 315-325.

11. Chabak L., Semenov V., Vedel Y. A New Non-Euclidean Proximal Method for Equilibrium Problems. In: Chertov O., Mylovanov T., Kondratenko Y., Kacprzyk J., Kreinovich V., Stefanuk V. (eds.) Recent Developments in Data Science and Intelligent Analysis of Information. ICDSIAI 2018. Advances in Intelligent Systems and Computing, vol. 836. Springer, Cham, 2019. P. 50-58.

12. Bauschke H. H., Combettes P. L. Convex Analysis and Monotone Operator Theory in Hilbert Spaces. Berlin, Heidelberg, New York: Springer, 2011. 408 p.

13. Malitsky Yu. V., Semenov V. V. An extragradient algorithm for monotone variational inequalities. Cybernetics and Systems Analysis. 2014. Vol. 50. P. 271-277. 
14. Semenov V. V. A Version of the Mirror descent Method to Solve Variational Inequalities. Cybernetics and Systems Analysis. 2017. Vol. 53. P. 234-243.

15. Semenov V. V. A variant of mirror descent method for solving variational inequalities. In: Polyakova, L. N. (ed.) Constructive Nonsmooth Analysis and Related Topics (dedicated to the memory of V. F. Demyanov). IEEE, 2017. P. 281-284. doi: https://doi.org/10.1109/CNSA.2017.7974011

16. Nomirovskii D. A., Rublyov V. V., Semenov V. V. Convergence of Two-Stage Method with Bregman Divergence for Solving Variational Inequalities. Cybernetics and Systems Analysis. 2019. Vol. 55. P. 359-368.

17. Gidel G., Berard H., Vincent P., Lacoste-Julien S. A Variational Inequality Perspective on Generative Adversarial Networks. arXiv:1802.10551. 2018.

18. Opial Z. Weak convergence of the sequence of successive approximations for nonexpansive mappings. Bull. Amer. Math. Soc. 1967. Vol. 73. P. 591-597.

Поступила: 02.04.2019 / Принята: 24.05.2019 